\title{
ASYMPTOTIC SHARPNESS OF BOUNDS ON HYPERTREES
}

\author{
YI LIN \\ LIYING KANG \\ Department of Mathematics \\ Shanghai University \\ Shanghai 200444, P.R. China \\ e-mail: linyi_sally@163.com \\ lykang@shu.edu.cn \\ AND \\ ERFANG SHAN ${ }^{1}$ \\ School of Management \\ Shanghai University \\ Shanghai 200444, P.R. China \\ e-mail: efshan@i.shu.edu.cn
}

\begin{abstract}
The hypertree can be defined in many different ways. Katona and Szabó introduced a new, natural definition of hypertrees in uniform hypergraphs and investigated bounds on the number of edges of the hypertrees. They showed that a $k$-uniform hypertree on $n$ vertices has at most $\left(\begin{array}{c}n \\ k-1\end{array}\right)$ edges and they conjectured that the upper bound is asymptotically sharp. Recently, Szabó verified that the conjecture holds by recursively constructing an infinite sequence of $k$-uniform hypertrees and making complicated analyses for it. In this note we give a short proof of the conjecture by directly constructing a sequence of $k$-uniform $k$-hypertrees.
\end{abstract}

Keywords: hypertree, semicycle in hypergraph, chain in hypergraph.

2010 Mathematics Subject Classification: 05C65.

\section{REFERENCES}

\footnotetext{
${ }^{1}$ Corresponding author.
} 
[1] C. Berge, Hypergraphs (Amsterdam, North-Holland, 1989).

[2] G.Y. Katona and P.G.N. Szabó, Bounds on the number of edges in hypertrees, Discrete Math. 339 (2016) 1884-1891.

doi:10.1016/j.disc.2016.01.004

[3] J. Nieminen and M. Peltola, Hypertrees, Appl. Math. Lett. 12 (1999) 35-38.

doi:10.1016/S0893-9659(98)00145-1

[4] B. Oger, Decorated hypertrees, J. Combin. Theory Ser. A 120 (2013) 1871-1905. doi:10.1016/j.jcta.2013.07.006

[5] P.G.N. Szabó, Bounds on the number of edges of edge-minimal, edge-maximal and l-hypertrees, Discuss. Math. Graph Theory 36 (2016) 259-278.

doi:10.7151/dmgt.1855

Received 30 April 2016

Revised 14 July 2016

Accepted 14 July 2016 